\title{
Unique neurophysiologic characteristics of the longest-living rodent: the naked mole rats
}

\author{
Meng Liang ${ }^{1}$, Bingying Du ${ }^{1}$, Rui Sun ${ }^{1}$, Ge Yin ${ }^{1}$, Cunxiu Fan ${ }^{1}$, and Xiaoying Bi ${ }^{1}$ \\ ${ }^{1}$ Affiliation not available
}

October 16, 2020

\begin{abstract}
Heterocephalus glaber or the naked mole rats(NMRs) belongs to the genus nude moles of the Bathyergidae family, which is the only kind of temperature changing mammal with true sociality in the world and shares $93 \%$ genetic homology with humans. Long-term subterranean burrowing living has led to NMRs gradually evolved a series of physiological characteristics that are significantly different from mammals living on the ground, such as vitamin D deficiency, sensory organs degeneration and extreme hypoxia tolerance. Despite the harsh living environment, NMRs are extremely long-lived. Their average lifespan is 5-7 times that of laboratorial mice of the same size, up to 30 years. These physiological characteristics of NMRs giving them a good applicable prospect when studying neurological related diseases such as pain, ischemic stroke, and Alzheimer's disease(AD). Here, we have reviewed the studies published on the neurophysiological characteristics of NMRs to provide reference for future research.
\end{abstract}

\section{Hosted file}

Naked mole rats.pdf available at https://authorea.com/users/367693/articles/487032-uniqueneurophysiologic-characteristics-of-the-longest-living-rodent-the-naked-mole-rats 